\title{
Chromatin insulation dynamics in glioblastoma: challenges and future perspectives of precision oncology
}

\author{
Borja Sesé* (1), Miquel Ensenyat-Mendez, Sandra Iñiguez, Pere Llinàs-Arias and Diego M. Marzese*
}

\begin{abstract}
Glioblastoma (GBM) is the most aggressive primary brain tumor, having a poor prognosis and a median overall survival of less than two years. Over the last decade, numerous findings regarding the distinct molecular and genetic profiles of GBM have led to the emergence of several therapeutic approaches. Unfortunately, none of them has proven to be effective against GBM progression and recurrence. Epigenetic mechanisms underlying GBM tumor biology, including histone modifications, DNA methylation, and chromatin architecture, have become an attractive target for novel drug discovery strategies. Alterations on chromatin insulator elements (IEs) might lead to aberrant chromatin remodeling via DNA loop formation, causing oncogene reactivation in several types of cancer, including GBM. Importantly, it is shown that mutations affecting the isocitrate dehydrogenase (IDH) 1 and 2 genes, one of the most frequent genetic alterations in gliomas, lead to genome-wide DNA hypermethylation and the consequent IE dysfunction. The relevance of IEs has also been observed in a small population of cancer stem cells known as glioma stem cells (GSCs), which are thought to participate in GBM tumor initiation and drug resistance. Recent studies revealed that epigenomic alterations, specifically chromatin insulation and DNA loop formation, play a crucial role in establishing and maintaining the GSC transcriptional program. This review focuses on the relevance of IEs in GBM biology and their implementation as a potential theranostic target to stratify GBM patients and develop novel therapeutic approaches. We will also discuss the state-of-the-art emerging technologies using big data analysis and how they will settle the bases on future diagnosis and treatment strategies in GBM patients.
\end{abstract}

\section{Introduction}

Glioblastoma (GBM) is the most aggressive type of primary brain tumor. The current standard-of-care (SOC) for patients with GBM includes a combination of surgical resection, adjuvant radiotherapy, and chemotherapy, mainly based on temozolomide (TMZ) [1, 2]. However, the prognosis of GBM patients remains dismal, with a median survival time of approximately 15 months and a recurrence rate of about $90 \%$ [3]. In addition to the

*Correspondence: borja.sese@ssib.es; diego.marzese@ssib.es Cancer Epigenetics Laboratory At the Cancer Cell Biology Group, Institut D'Investigació Sanitària Illes Balears (IdISBa), Carretera de Valldemosa 79, S Building, 1st Floor, 07120 Palma de Mallorca, Spain limited benefit in survival, SOC treatments cause significant morbidity involving neurological deficits. Formerly known as glioblastoma multiforme, the term "multiforme" reflects a robust heterogeneous variety of cell types coexisting within the tumor. Each cell type exhibits a particular molecular profile, leading to different degrees of therapy resistance among its tumor cell population [4, 5]. The detection and characterization of such intratumor heterogeneity are of great value to the clinical diagnosis and management of this disease. GBM can develop rapidly as a de novo brain tumor (primary GBM) in more than $90 \%$ of cases [6]. To a lesser extent, these tumors can originate from previous lower-grade diffuse gliomas (secondary GBM). Although these are histologically 
indistinguishable, they present distinct genetic and epigenetic signatures that allow their identification.

Recent molecular and computational biology improvements allowed the identification of novel targetable molecular mechanisms in GBM. Gene- and gene pathway-centered approaches have generated a myriad of data about GBM mechanisms contributing to invasion, progression, unlimited replication, maintenance, and drug resistance [7-9]. However, to date, the contribution of these scientific advances to the clinical management of GBM patients remains insufficient. The limited improvements in the clinical outcomes reflect the inherent multimolecular-level, omics-scale complexity that defines GBM etiology and pathology. The absence of effective therapeutic management represents an inherent challenge to treat GBM. Taken together, these issues motivate the need for alternative approaches to better understand and disentangle the integrative molecular alterations underpinning the aggressive and treatment-resistant phenotype of GBM.

Genetic and epigenetic alterations on insulator elements (IEs), an essential type of cis-regulatory element involved in enhancer-promoter interactions, have been recently found in cancer cells [10]. In particular, dysfunctional IEs result in aberrant chromatin conformation and the consequent oncogene activation [11]. Therefore, this review covers the most recent findings on the role of IEs in GBM and the potential effects on gene network regulation. At the same time, it provides an overview of the chromatin architecture and IEs in glioma stem cells (GSCs) and its potential translation into novel patient-centered diagnosis, prognosis, and therapeutic applications.

Role of insulator elements in chromatin organization, gene isolation, and concomitant gene expression

The essential role of IEs is the compartmentalization of chromatin into structural and functional units known as Topologically Associating Domains (TADs). TADs are megabase-size regions formed and maintained by the architectural protein CCCTC-binding factor (CTCF) that restrict chromatin interactions within themselves compared to neighboring genomic regions [12]. TADs can act as a single insulated region or as a multidomain structure containing several insulated segments, often referred to as sub-TADs. Both TADs and sub-TADs are organized into distinct units of globular conformation, forming DNA loops that confer physical isolation of genes confined within a TAD structure that prevents their interaction with regulatory regions located outside of the TAD [12, 13]. It has been proposed that TAD formation follows the "loop extrusion" model where CTCF and cohesin are the major players [14-17]. In this model, the cohesin complex translocates onto chromatin. It travels along the DNA molecule, extruding a chromatin loop on its way until it reaches a CTCF bound to an inward-oriented CTCF site, blocking further chromatin extrusion.

IEs regulate gene expression via loop formation in a position-dependent manner [18]. CTCF-cohesin anchored loops can facilitate enhancer-promoter interactions when flanking both elements to constrain the enhancer function within the DNA loop, resulting in a structural unit known as an "insulated neighborhood" [19] (Fig. 1a). Conversely, IEs can also block enhancerpromoter interactions by capturing a gene promoter inside a chromatin loop, unable to reach its former enhancer element (EE). This type of IE is known as "enhancer-blocking elements" and can only occur if the insulator sequence is placed between the $\mathrm{EE}$ and the gene promoter (Fig. 1b). Also, IEs can separate active chromatin (euchromatin) from repressive chromatin regions (heterochromatin), independently of CTCF-loop formation. This latter type of IE is known as "barrier elements," and their mechanism of action consists of recruiting histone modifying enzymes, acting as a physical barrier to protect against the linear spread of heterochromatin into euchromatin regions, and vice versa [20] (Fig. 1c). All these IE functions are notably important in sub-TADs, where they grant a more dynamic regulatory control over genes located within the loop structure [21]. While TADs are highly conserved among mammals and different tissues, sub-TADs are more variable. They tend to be associated with changes in response to cellular signals, gene activity and allow for cell-specific functions [22, 23]. These observations suggest that sub-TADs allow finetuned control of the gene expression during specific transcriptional programs such as cell differentiation [24].

\section{Aberrant chromatin insulation function in cancer}

Alterations in TAD assembly and high-order chromatin organization are often associated with multiple developmental defects and diseases. While many efforts have been made to study transcriptional regulatory elements in cancer, most of these focused on promoters and EEs. Regarding IEs, loss of CTCF boundaries may disrupt insulated neighborhoods containing oncogenes, thus becoming reactivated [25]. Alternatively, alterations in gene expression could influence and reshape chromatin loops to gain tumor-related traits, as shown in several types of cancer [26]. Aberrant insulator function could be associated with missense mutations in the CTCF coding region and CTCF binding sites (CBSs), leading to disrupted loop-formation activity and gene expression dysregulation [27-29]. Supporting the relevance of chromatin insulation in cancer, Liu et al. identified 21 IE mutations as putative drivers of multiple cancer types 

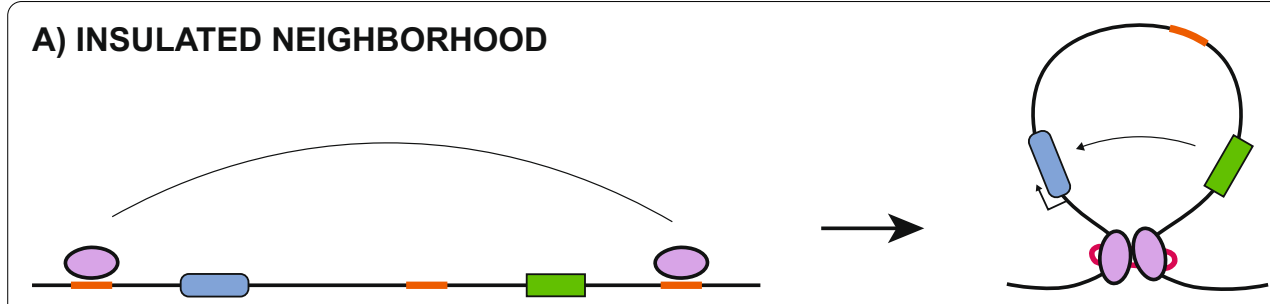

B) ENHANCER-BLOCKING ELEMENTS
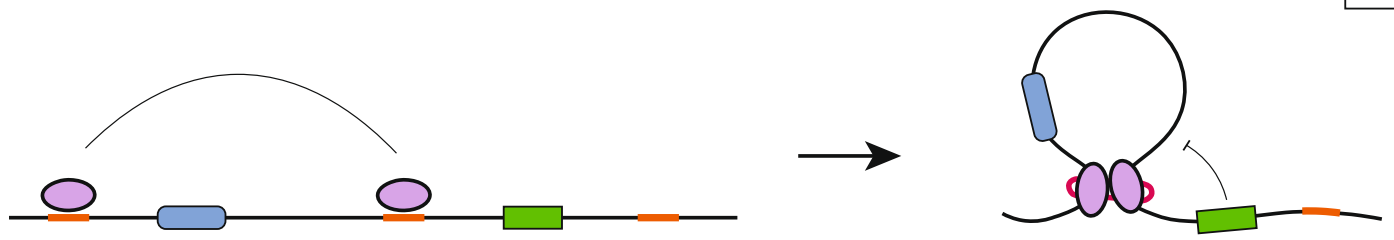

C) BARRIER ELEMENTS

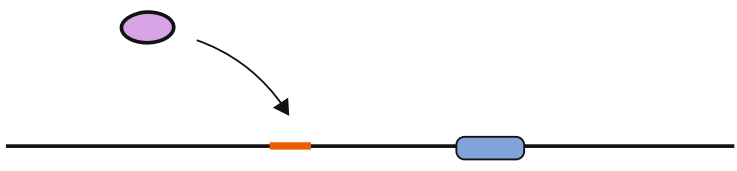

EUCHROMATIN

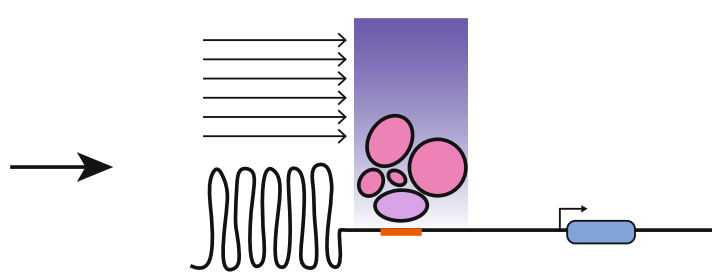

HETEROCHROMATIN EUCHROMATIN

Fig. 1 Insulator elements mechanism of action. a Insulator flanking a transgene and an enhancer element generates an insulated neighborhood via CTCF-loop formation, favoring enhancer-promoter interactions within the DNA loop. $\mathbf{b}$ Enhancer-blocking elements result from insulators between a transgene and an enhancer, leaving the enhancer outside the DNA loop, blocking its interaction with the transgene promoter. $\mathbf{c}$ Insulators acting as barrier elements prevent the spread of heterochromatin by recruiting histone-modifying enzymes (HME) to preserve a transcriptionally active euchromatin state

[10]. In addition to somatic mutations, CBSs could also be affected by epigenetic modifications. The hypomethylation of CBS promotes a higher occupancy by CTCF, resulting in de novo formation of TAD boundaries causing deregulation of gene expression often linked to tumor suppressor silencing [30]. On the contrary, hypermethylation of CBS reduces the recruitment of CTCF, which is followed by the disruption of TAD domains and, in many cases, leads to aberrant promoter-enhancer interactions known as "enhancer adoption" [31]. This enhancer adoption event is frequently related to oncogene activation [32].

\section{Dynamic function of chromatin insulation in glioblastoma} Given GBM intratumor heterogeneity, a significant effort has been made to search for epigenetic features to stratify these tumors into subcategories for accurate diagnosis and treatment $[33,34]$. The major genomic alterations described in GBM are the mutations affecting the isocitrate dehydrogenase (IDH) 1 and 2 genes. IDH enzymes are best known for playing an essential role in several metabolic processes. As such, recurrent mutations of these genes are present in various human malignancies [35]. While IDH wild-type (IDH-wt) enzymes convert isocitrate into $\alpha$-ketoglutarate $(\alpha-K G)$ as part of the Krebs cycle, IDH mutant (IDH-mt) enzymes lose their affinity for isocitrate and convert $\alpha$-KG into D-2-hydroxyglutarate (D-2-HG) [36]. Accumulation of D-2-HG leads to severe epigenetic alterations by blocking the activity of ten-eleven translocation methylcytosine dioxygenase (TET), a family of enzymes involved in DNA demethylation [37]. Consequently, IDH mutations result in widespread $\mathrm{CpG}$ island methylation, a particular signature known as glioma $\mathrm{CpG}$ island methylator phenotype (G-CIMP). Given the dramatic clinical and molecular differences associated with the G-CIMP, GBM is classified into two distinctive categories: IDH-mt and IDH-wt [38]. IDH-mt GBMs represent less than 10\% of GBM cases, frequently associated with younger individuals, and typically correspond with secondary GBM. On the other hand, IDH-wt GBMs comprise most GBM cases, mostly primary tumors, often diagnosed in elderly patients with 
a significantly worse prognosis than IDH-mt GBM [39]. Inevitably, genome-wide DNA hypermethylation has a notorious impact on chromatin insulation by compromising IE functionality. Flavahan et al. [11] reported that many CTCF sites were lost in IDH-mt GBM. A wellstudied case of insulator dysfunction in IDH-mt occurs after hypermethylation of CBS and the consequent disruption of TAD boundaries, enabling the interaction of a constitutive EE with the promoter region of the receptor tyrosine kinase platelet-derived growth factor receptor $\mathrm{A}$ (PDGFRA) gene (enhancer adoption), a well-described glioma oncogene [11]. However, most likely, many other oncogenic events in GBM are deregulated due to epigenetic alterations and chromatin insulation loss. Expanding our understanding of how IEs reshape chromatin and lead to oncogenic reactivation in GBM may uncover novel therapeutic targets to improve the diagnosis and targeted treatments.

\section{Insulator elements influence gene expression in glioma stem cells}

Malignant tumors consist of heterogeneous cell populations, including a small subset called cancer stem cells (CSCs), or tumor-initiating cells, which are thought to initiate tumor formation, promote metastasis, and grant resistance to therapy [40-42]. In GBM, these cells are known as GSCs and have become a key target for therapeutic developments with the aim to eliminate tumor therapy-resistant traits and relapse [43-46]. GSCs must activate several signaling pathways such as $\mathrm{NOTCH}$, bone morphogenetic protein (BMP), wingless-related integration site (WNT), epidermal growth factor (EGF), and sonic hedgehog $(\mathrm{SHH})$ to maintain an undifferentiated state often associated with stemness and required for self-renewal and rapid differentiation upon tumor progression in CSCs [47]. This provides GSCs a cellular plasticity capable of generating all the different cell types found in the tumor bulk. Also, GSCs present genomic regions where active histone marks (H3K4me3) coexist with repressive histone modifications (H3K27me3), the so-called bivalent domains, often associated with repressed lineage-specific genes to maintain an undifferentiated state [48, 49]. A recent work published by Hall et al. [49] has shown that bivalent regions within GBM primary tumors are part of a highly interconnected network under the influence of WNT, SHH, and HOX pathways, commonly associated with embryonic development. Thus, a subset of transcription factors (TFs) may be responsible for establishing a permissive chromatin architecture that maintains stemness through several cell divisions in GSCs, which, in turn, confers aggressive traits, including tumor progression and drug resistance.
A proper chromatin assembly into structural subunits is required to coordinate specific gene expression programs to establish and maintain GSC stemness. GSCs present a specific subset of large clusters of EEs known as super-enhancers (SEs) that drive a robust transcriptional program determined by core TFs [50]. A recent study conducted by Johnston et al. [51] revealed that genes interacting with SEs within a DNA loop are highly expressed in GSCs. Moreover, some of these loops containing SEs seem to be GSC-specific as they are strongly conserved among different GSC lines. In this same work, the authors also showed that structural variants in the GSC genome cause rare long-distance loops resulting in de novo SE-promoter interactions. Most of these gene sets, highly connected through extensive chromatin looping, play a significant role in brain tumors and stem cell biology. Also, an enrichment of TFs regulated by GSCspecific SEs is associated with shorter survival of GBM patients, suggesting an essential role of SEs mediating the transcriptional regulatory program behind the maintenance of a GSC phenotype [50]. These data highlight the importance of IEs and TAD formation as a key regulatory process to assemble the GSC epigenome.

Genome architecture and chromatin insulation are essential to maintain cells in an undifferentiated state, propitiate cell plasticity, and coordinate differentiation programs into cell lineages. Differentiation of embryonic stem cells (ESCs) into neural precursors has been correlated with a gain of structural loops and enhanced binding of CTCF and cohesin leading to durable insulation between chromatin boundaries, limiting the enhancerpromoter interaction to the detriment of the activation of developmental genes [52]. Similar to ESCs, GSCs also exhibit a more accessible genome-wide chromatin state with a global loss of repressive (H3K9me3) and gain of active (H3K9ac) histone modification marks [53]. Based on these findings, GSCs may present a similar behavior to ESCs, where a permissive chromatin state is required to sustain self-renewal, and the structural loop rearrangements are needed to reinforce chromatin boundaries and give rise to differentiated progeny that will constitute the tumor cell mass (Fig. 2). For example, a neural stem cell model for low-grade astrocytoma containing $I D H 1 / 2$ gene mutations caused the loss of CTCF binding and the subsequent dissociation of the sex determining region y-box 2 (SOX2) promoter-enhancer interaction [54]. This alteration resulted in an impaired ability to differentiate in vivo due to chromatin loop disruption, thus increasing its self-renewal and invasiveness capacities. Taken together, IEs could emerge as a new therapeutic target to prevent tumor growth in GBM. However, further analyses are required to elucidate the impact of IEs and chromatin architecture in GSCs on drug resistance, 


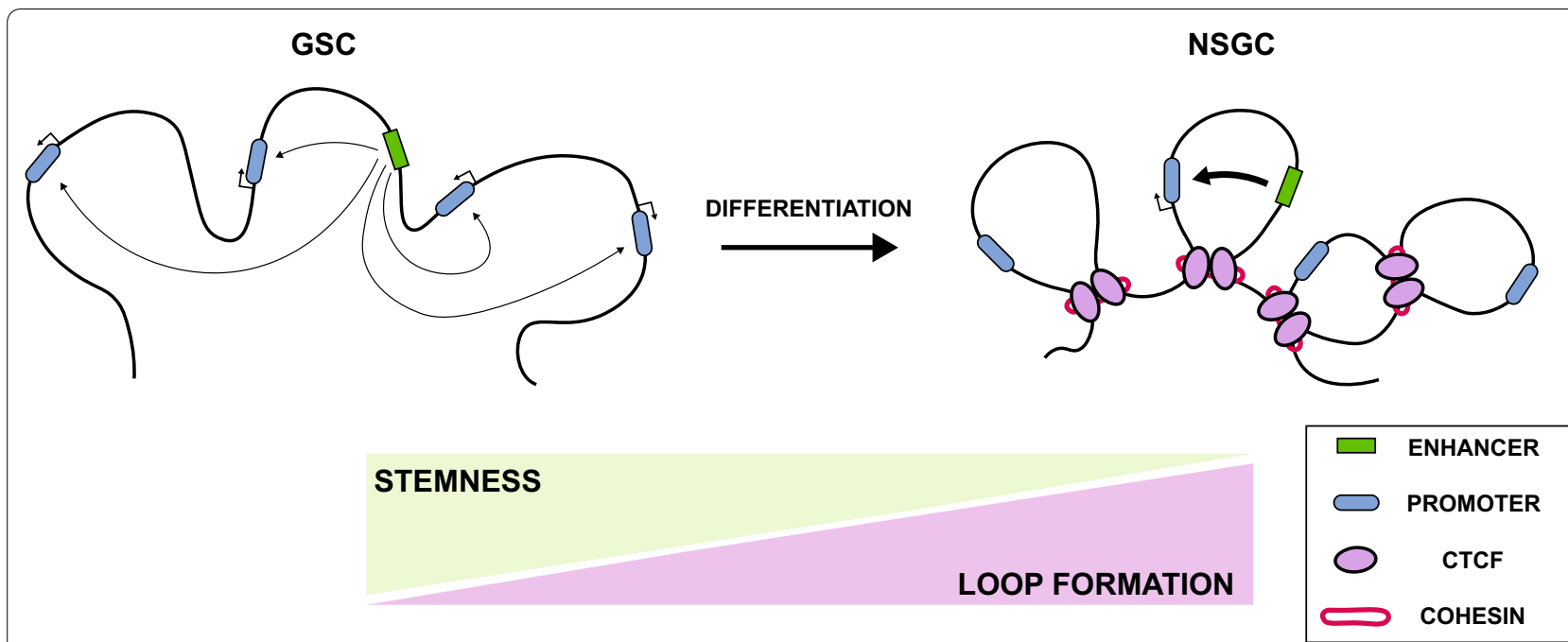

Fig. 2 Chromatin loop formation in GSC differentiation. GSCs present a more relaxed and accessible chromatin state with permissive interactions between enhancers and promoters among different domains. During GSC differentiation, loss of stemness in non-stem glioma cells (NSGC) correlates with increased chromatin-loop formation, adopting a more restricted chromatin conformation with strong domain boundaries and limited enhancer-promoter interactions

tumor survival, and relative toxicity and off-target effects of its inhibition.

\section{How to modulate epigenomic alterations in glioblastoma? Technology for druggability testing}

GBM treatment remains a challenge as current therapies can only improve median prognosis by a few months. Epigenetic modulators have become an attractive therapeutic target for drug discovery to efficiently regulate cancer growth and progression. Numerous studies on histone deacetylases inhibitors have shown promising results in various cancers $[55,56]$, including GBM [57-61]. Following this research line, there is a particular interest in identifying novel compounds to target cancer regulatory elements. The JQ1, a bromodomain and extra-terminal domain (BET) domain inhibitor, has been shown to downregulate the expression of wellknown oncogenes such as the MYC proto-oncogene, bHLH transcription factor $(M Y C)$ and B-cell lymphoma 2 (BLC2) by blocking bromodomain-containing protein 4 (BRD4) binding at associated SE regions in different cancers $[62,63]$. Combined with conventional chemotherapy agents, BET inhibitors showed a significant anti-tumor effect in GBM xenograft models [64]. However, the efficacy of BET inhibitors in clinical trials remains untested. Recent studies have revealed the importance of architectural proteins in cancer treatment and how aberrant CTCF function can contribute to a drug-resistant phenotype $[26,65]$. In a recent work, Kantidze et al. discovered curaxins, a novel class of DNA intercalating agents that promote the dissociation of CTCF from its binding site causing chromatin loop disorganization in tumor cells $[66,67]$. These modifications resulted in transcriptional suppression of several MYC family oncogenes after losing their long-distance interactions with their respective SEs. These findings support the potential of epigenetic therapies targeting GBM-specific IEs involved in tumorigenesis and therapy resistance. This strategy is currently being tested in phase I clinical trial for patients with cutaneous melanoma or sarcoma (ClinicalTrials. gov, NCT03727789). Nevertheless, the implementation of this strategy is still non-genome site specific. A novel clustered regularly interspaced short palindromic repeats (CRISPR)-based approach consisting of a catalytically inactive CRISPR-associated protein 9 (dCas9) fused with the Krüppel-associated box (KRAB) transcriptional repressor and DNA-methyltransferase 3A (DNMT3A) can selectively disrupt CTCF recruitment to its CBS, allowing to study the role of IEs in a locus-specific manner [68]. Although Cas9-fused proteins are far from being used to treat GBM, the first-ever clinical trial to directly deliver CRISPR-Cas9 in the body has recently begun recruiting patients with retinal diseases (ClinicalTrials.gov, NCT03872479), bringing this technology one step closer for clinical applications. Forward-thinking, a potential strategy would involve restoring chromatin conformation by targeting IEs involved in enhancer-promoter interactions to prevent (1) oncogene activation, (2) tumor suppressor gene silencing, and (3) interaction of SEs with stemness genes in GSCs. However, despite the promising potential of drugs targeting tumor cells genome-wide organization, further studies would be 
required to anticipate and prevent undesired outcomes. Therefore, a better understanding of chromatin insulators and their role in dynamic chromatin organization is a crucial initial step toward a more effective therapeutic combination and personalized anti-GBM therapy.

\section{Impact of insulator elements on glioblastoma precision oncology}

The next frontier in the clinical management of GBM is the implementation of precision medicine models to generate biomarkers for early detection, identification of patient-tailored treatments, and personalized follow-up. Advances in GBM epigenetics, particularly IEs modulating chromatin conformation, could provide additional information on current multi-omics profiling. Epigenetic alterations in GBM are currently employed for response to TMZ prediction (O-6-methylguanine-DNA methyltransferase (MGMT) gene promoter hypermethylation) [69], prognosis estimation (G-CIMP) [70], and molecular stratification (GBM molecular subtype classification) [5]. Advances in integrating the patient's and tumor's genetic makeups with epigenome-wide DNA methylation profiles, chromatin conformation, and TF occupancy to generate gene regulatory circuits active in each patient will improve our ability to stratify patients and improve clinical management (Fig. 3). These changes will advance our ability to build accurate models capable of determining the best available treatment and improve the clinical management of this deadly disease. As recently proposed, the implementation of systems biology approaches to integrate clinical with multi-omics data, including epigenetics, provides the basis to design $N$-of-1 precision medicine treatments [71]. Examples of these advances involve the repurposing of drugs and treatments that have shown poor results in unselected cohorts of GBM patients. Such an endeavor will be appeased by emerging technologies capable of profiling epigenetic landscapes with minimum tissue requirements. The most remarkable improvements in this area involve the capabilities to perform dual chromatin accessibility and DNA methylation profiling [72, 73], long-read sequencing that allows profiling DNA modifications beyond 5-methylcytosine, and chromatin accessibility [72, 74-76], and its applications to singlecell samples.

Regarding the study of chromatin architecture, many techniques have been developed during the last years. $3 \mathrm{D}$ chromatin is mainly assessed through three different approaches: (1) by imaging, which includes DNAFluorescence in Situ Hybridization (DNA-FISH) and its derivatives; (2) using chromatin conformation capture (3C)-derived techniques, which require the ligation of DNA fragments; and (3) performing ligation-free methods. The advantages and limitations of these techniques are greatly discussed in a recent review by Kempfer et al. [77]. The rise of the studies of 3D chromatin may increase our understanding of GBM biology. However, many of the current techniques require large amounts of cells, representing an unsolved limitation. To overcome this restriction, the techniques that may be translated to the bedside for GBM are genome architecture mapping (GAM) and Capture $\mathrm{Hi}-\mathrm{C}$, which require less than $10^{5}$ cells. GAM is based on laser microdissection on sucroseembedded cells, followed by DNA sequencing [78]. Capture $\mathrm{Hi}-\mathrm{C}$ is a $3 \mathrm{C}$-based method where the $3 \mathrm{C}$ library can be enriched for targets of interest. This approach has been used to detect interactions to promoter regions genome-wide [79], but it can also be performed to detect interactions between IEs.

On the other hand, genetic variations affecting noncoding genomic regions are starting to find functional relevance due to the integration of Genome-Wide Association Studies (GWAS) datasets with GBM epigenetic profiles [80]. For example, mutations in the promoter region of the TERT gene modify the binding affinity of the cAMP response element-binding protein (CREB) transcription factor and lead to an enhanced expression of the telomerase gene in several types of cancer [81, 82]. Today, this non-coding alteration is part of the genetic changes considered for GBM classification [83]. Similarly, we believe that dysfunctional IEs could be considered potential theranostic markers for personalized treatments in GBM. A significant milestone to successfully apply this strategy is to comprehensively overlay mutation signatures, gene expression, and clinical information with master TF regulators of the epigenomic dynamics. Thus, key TFs and microRNA orchestrating transcriptional regulatory networks (TRN) in gliomas have been unraveled thanks to the development and application of a platform known as the GBM SYstems Genetics Network AnaLysis pipeline (gbmSYGNAL) [84]. This pipeline has shown that TRN structures can be integrated with data from currently-approved cancer drugs to identify patient-specific novel and synergistic therapeutic interactions. This pioneering development is just the start of the upcoming era of GBM precision medicine aided by epigenomic networks.

\section{Conclusions}

Although TAD formation and its role in regulating gene expression have been widely studied over the last years, the mechanisms on how enhancers preferentially interact with their target genes are still not fully understood. As a result, alterations of non-coding regulatory elements associated with tumorigenesis, and IEs in particular, have been largely underestimated. Given the relevance of these high-order chromatin alterations 


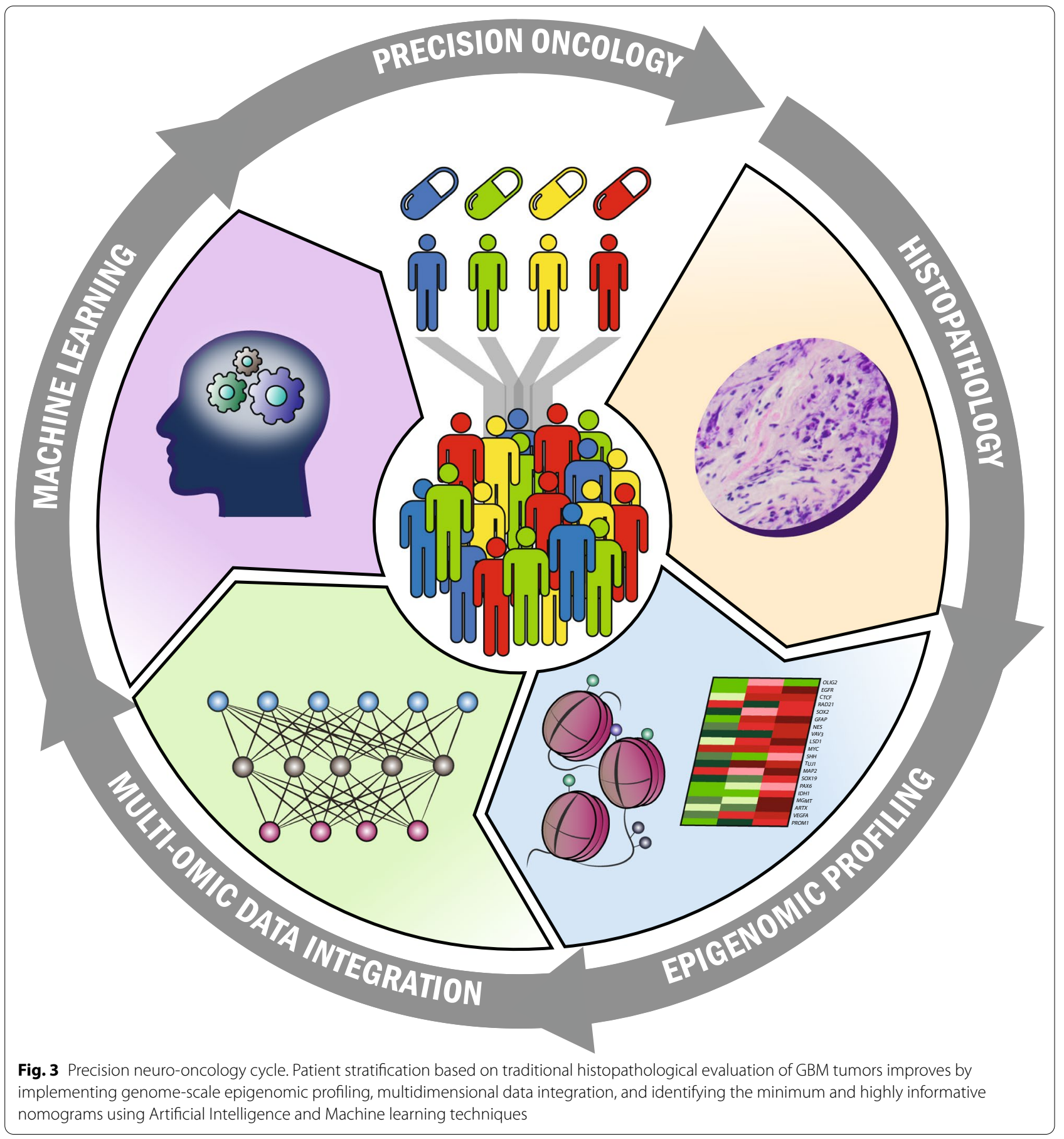

in driving oncogenic programs, continued efforts to uncover IE regulatory mechanisms are required. In GBM, an in-depth look at how IEs reshape chromatin topology to achieve a malignant phenotype will be paramount to find new therapeutic strategies against this devastating type of tumor. The road to integrating epigenomics into clinical decision-making algorithms for patients with GBM will be paved with progress in computing capabilities and enhanced analytical algorithms. Technological advances are needed to reduce the complexity of widespread aberrant chromatin insulation and its impact on long-range interactions among genomic regulatory elements. Computer science leading the incorporation of artificial intelligence into 
clinical nomograms to generate integrative systems for medicine is changing our approaches to analyze largescale and complex datasets. Machine learning algorithms are already changing the field of cancer diagnosis and prognosis by exploring diverse data types, including imaging, histology, and multi-omics, to efficiently classify various clinically relevant GBM traits [85-88]. These advances will undoubtedly bring novel and more personalized diagnostic and therapeutic alternatives for patients with GBM.

\begin{abstract}
Abbreviations
GBM: Glioblastoma; IE: Insulator element; IDH: Isocitrate dehydrogenase; IDH-wt: Isocitrate dehydrogenase wild-type; IDH-mt: Isocitrate dehydrogenase mutant; GSC: Glioma stem cells; SOC: Standard-of-care; TMZ: Temozolomide; TAD: Topologically Associating Domains; CTCF: CCCTC-binding factor; EE: Enhancer element; CBS: CTCF binding sites; a-KG: a-Ketoglutarate; D-2-HG: D-2-hydroxyglutarate; TET:Ten-eleven translocation methylcytosine dioxygenase; G-CIMP: Glioma CpG island methylator phenotype; PDGFRA: Plateletderived growth factor receptor A; CSC: Cancer stem cell; BMP: Bone morphogenetic protein; WNT:Wingless-related integration site; EGF: Epidermal growth factor; SHH: Sonic hedgehog; H3K4me3: Histone H3 lysine K4 trimethylation; H3K27me3: Histone H3 lysine K27 trimethylation; H3K9me3: Histone H3 lysine K9 trimethylation; H3K9ac: Histone H3 lysine K9 acetylation; TF: Transcription factor; SE: Super-enhancer; ESC: Embryonic stem cell; SOX2: Sex-determining region y-box 2; BET: Bromodomain and extraterminal; BLC2: B-cell lymphoma 2; BRD4: Bromodomain-containing protein 4; MYC: MYC proto-oncogene, bHLH transcription factor; CRISPR: Clustered regularly interspaced short palindromic repeats; Cas9: CRISPR-associated protein 9; KRAB: Krüppel-associated box; DNMT3A: DNA-methyltransferase 3A; MGMT: O-6-methylguanine-DNA methyltransferase; 3D: Three-dimensional; DNA-FISH: DNA-fluorescence in situ hybridization; GAM: Genome architecture mapping; 3C: Chromosome conformation capture; Hi-C: High-throughput chromosome conformation capture; TERT: Telomerase reverse transcriptase; CREB: CAMP response elementbinding; TRN: Transcriptional regulatory networks; gbmSYGNAL: Glioblastoma systems genetics network analysis pipeline; HME: Histone-modifying enzymes; NSGC: Non-stem glioma cells.
\end{abstract}

\section{Acknowledgements}

Not applicable.

\section{Authors' contributions}

$\mathrm{BS}$ and $\mathrm{DM}$ contributed to the conceptualization of the review. BS, ME, and $\mathrm{SI}$ researched the bibliography and contributed to writing the original draft preparation. $\mathrm{BS}, \mathrm{ME}, \mathrm{SI}, \mathrm{PL}$, and $\mathrm{DM}$ wrote and reviewed the manuscript. $\mathrm{BS}, \mathrm{SI}$, and DM contributed to the illustration design. All authors read and approved the final version of the manuscript.

\section{Funding}

This work was supported by the Instituto de la Salud Carlos III Miguel Servet Project (\#CP17/00188) and AES 2019 (\#PI19/01514) co-funded by European Regional Development Fund "A way to make Europe", the Institut d'Investigació Sanitària Illes Balears (FOLIUM program), the Govern de les Illes Balears (Margalida Comas program), the Fundación Francisco Cobos, and the Asociación Española Contra el Cancer.

\section{Availability of data and materials}

Not applicable.

\section{Declarations}

Ethics approval and consent to participate Not applicable.
Consent for publication

Not applicable.

\section{Competing interests}

The authors in this article declare that they have no competing interests.

Received: 3 April 2021 Accepted: 23 July 2021

Published online: 31 July 2021

\section{References}

1. Lau D, Magill ST, Aghi MK. Molecularly targeted therapies for recurrent glioblastoma: current and future targets. Neurosurg Focus. 2014;37:E15.

2. Thakkar JP, Dolecek TA, Horbinski C, Ostrom QT, Lightner DD, BarnholtzSloan JS, et al. Epidemiologic and molecular prognostic review of glioblastoma. Cancer Epidemiol Biomark Prev Publ Am Assoc Cancer Res Cosponsored Am Soc Prev Oncol. 2014;23:1985-96.

3. Weller M, Cloughesy T, Perry JR, Wick W. Standards of care for treatment of recurrent glioblastoma-are we there yet? Neuro-Oncol. 2013;15:4-27.

4. Brennan CW, Verhaak RGW, McKenna A, Campos B, Noushmehr H, Salama SR, et al. The somatic genomic landscape of glioblastoma. Cell. 2013;155:462-77.

5. Verhaak RGW, Hoadley KA, Purdom E, Wang V, Qi Y, Wilkerson MD, et al. Integrated genomic analysis identifies clinically relevant subtypes of glioblastoma characterized by abnormalities in PDGFRA, IDH1, EGFR, and NF1. Cancer Cell. 2010;17:98-110.

6. Ohgaki H, Kleihues P. The definition of primary and secondary glioblastoma. Clin Cancer Res. 2013;19:764-72.

7. Fan Q-W, Cheng CK, Gustafson WC, Charron E, Zipper P, Wong RA, et al. EGFR phosphorylates tumor-derived EGFRvIll driving STAT3/5 and progression in glioblastoma. Cancer Cell. 2013;24:438-49.

8. Keunen O, Johansson M, Oudin A, Sanzey M, Rahim SAA, Fack F, et al. Anti-VEGF treatment reduces blood supply and increases tumor cell invasion in glioblastoma. Proc Natl Acad Sci U S A. 2011;108:3749-54.

9. Keunen O, Taxt T, Grüner R, Lund-Johansen M, Tonn J-C, Pavlin T, et al. Multimodal imaging of gliomas in the context of evolving cellular and molecular therapies. Adv Drug Deliv Rev. 2014;76:98-115.

10. Liu EM, Martinez-Fundichely A, Diaz BJ, Aronson B, Cuykendall T, MacKay $\mathrm{M}$, et al. Identification of cancer drivers at CTCF insulators in 1,962 whole genomes. Cell Syst. 2019;8:446-455.e8.

11. Flavahan WA, Drier Y, Liau BB, Gillespie SM, Venteicher AS, StemmerRachamimov AO, et al. Insulator dysfunction and oncogene activation in IDH mutant gliomas. Nature. 2016;529:110-4.

12. Bintu B, Mateo LJ, Su J-H, Sinnott-Armstrong NA, Parker M, Kinrot S, et al. Super-resolution chromatin tracing reveals domains and cooperative interactions in single cells. Science. 2018;362:eaau1783.

13. Miron E, Oldenkamp R, Brown JM, Pinto DMS, Xu CS, Faria AR, et al. Chromatin arranges in chains of mesoscale domains with nanoscale functional topography independent of cohesin. Sci Adv. 2020;6:eaba8811.

14. Guo Y, Xu Q, Canzio D, Shou J, Li J, Gorkin DU, et al. CRISPR inversion of CTCF sites alters genome topology and enhancer/promoter function. Cell. 2015;162:900-10.

15. de Wit E, Vos ES, Holwerda SJ, Valdes-Quezada C, Verstegen MJ, Teunissen $\mathrm{H}$, et al. CTCF binding polarity determines chromatin looping. Mol Cell. 2015;60:676-84.

16. Sanborn AL, Rao SS, Huang SC, Durand NC, Huntley MH, Jewett Al, et al. Chromatin extrusion explains key features of loop and domain formation in wild-type and engineered genomes. Proc Natl Acad Sci U A. 2015;112:E6456-65

17. Fudenberg G, Imakaev M, Lu C, Goloborodko A, Abdennur N, Mirny LA. Formation of chromosomal domains by loop extrusion. Cell Rep. 2016;15:2038-49.

18. Valenzuela L, Kamakaka RT. Chromatin insulators. Annu Rev Genet. 2006:40:107-38.

19. Hnisz D, Day DS, Young RA. Insulated neighborhoods: structural and functional units of mammalian gene control. Cell. 2016;167:1188-200.

20. Ali T, Renkawitz R, Bartkuhn M. Insulators and domains of gene expression. Curr Opin Genet Dev. 2016;37:17-26.

21. Matthews BJ, Waxman DJ. Computational prediction of CTCF/cohesinbased intra-TAD loops that insulate chromatin contacts and gene 
expression in mouse liver. Elife [Internet]. 2018;7. http://www.ncbi.nlm. nih.gov/pubmed/29757144.

22. Hanssen LLP, Kassouf MT, Oudelaar AM, Biggs D, Preece C, Downes DJ, et al. Tissue-specific CTCF-cohesin-mediated chromatin architecture delimits enhancer interactions and function in vivo. Nat Cell Biol. 2017;19:952-61.

23. Vietri Rudan M, Barrington C, Henderson S, Ernst C, Odom DT, Tanay $\mathrm{A}$, et al. Comparative Hi-C reveals that CTCF underlies evolution of chromosomal domain architecture. Cell Rep. 2015;10:1297-309.

24. Narendra V, Bulajic M, Dekker J, Mazzoni EO, Reinberg D. CTCF-mediated topological boundaries during development foster appropriate gene regulation. Genes Dev. 2016;30:2657-62.

25. Hnisz D, Weintraub AS, Day DS, Valton A-L, Bak RO, Li CH, et al. Activation of proto-oncogenes by disruption of chromosome neighborhoods. Science. 2016;351:1454-8.

26. Debruyne DN, Dries R, Sengupta S, Seruggia D, Gao Y, Sharma B, et al. BORIS promotes chromatin regulatory interactions in treatment-resistant cancer cells. Nature. 2019;572:676-80.

27. Filippova GN, Qi C-F, Ulmer JE, Moore JM, Ward MD, Hu YJ, et al. Tumorassociated zinc finger mutations in the CTCF transcription factor selectively alter tts DNA-binding specificity. Cancer Res. 2002;62:48-52.

28. Guo YA, Chang MM, Huang W, Ooi WF, Xing M, Tan P, et al. Mutation hotspots at CTCF binding sites coupled to chromosomal instability in gastrointestinal cancers. Nat Commun. 2018;9:1520.

29. Katainen R, Dave K, Pitkänen E, Palin K, Kivioja T, Välimäki N, et al. CTCF/ cohesin-binding sites are frequently mutated in cancer. Nat Genet. 2015:47:818-21.

30. Taberlay PC, Achinger-Kawecka J, Lun AT, Buske FA, Sabir K, Gould $\mathrm{CM}$, et al. Three-dimensional disorganization of the cancer genome occurs coincident with long-range genetic and epigenetic alterations. Genome Res. 2016:26:719-31.

31. Lettice LA, Daniels S, Sweeney E, Venkataraman S, Devenney PS, Gautier $P$, et al. Enhancer-adoption as a mechanism of human developmental disease. Hum Mutat. 2011;32:1492-9.

32. Kaiser VB, Semple CA. When TADs go bad: chromatin structure and nuclear organisation in human disease. F1000 Res. 2017;6:314.

33. Capper D, Jones DTW, Sill M, Hovestadt V, Schrimpf D, Sturm D, et al. DNA methylation-based classification of central nervous system tumours. Nature. 2018;555:469-74.

34. Paul Y, Mondal B, Patil V, Somasundaram K. DNA methylation signatures for 2016 WHO classification subtypes of diffuse gliomas. Clin Epigenetics. 2017;9:32

35. Dang L, Yen K, Attar EC. IDH mutations in cancer and progress toward development of targeted therapeutics. Ann Oncol. 2016;27:599-608.

36. Dang L, White DW, Gross S, Bennett BD, Bittinger MA, Driggers EM, et al. Cancer-associated IDH1 mutations produce 2-hydroxyglutarate. Nature. 2009;462:739-44

37. Xu W, Yang H, Liu Y, Yang Y, Wang P, Kim S-H, et al. Oncometabolite 2-hydroxyglutarate is a competitive inhibitor of a-ketoglutaratedependent dioxygenases. Cancer Cell. 2011;19:17-30.

38. Louis DN, Perry A, Reifenberger G, von Deimling A, Figarella-Branger D, Cavenee WK, et al. The 2016 world health organization classification of tumors of the central nervous system: a summary. Acta Neuropathol (Berl). 2016;131:803-20.

39. Nobusawa S, Watanabe T, Kleihues P, Ohgaki H. IDH1 mutations as molecular signature and predictive factor of secondary glioblastomas. Clin Cancer Res. 2009;15:6002-7.

40. Clarke MF, Dick JE, Dirks PB, Eaves CJ, Jamieson CHM, Jones DL, et al. Cancer stem cells - perspectives on current status and future directions: AACR workshop on cancer stem cells. Cancer Res. 2006;66:9339-44.

41. Gupta PB, Onder TT, Jiang G, Tao K, Kuperwasser C, Weinberg RA, et al. Identification of selective inhibitors of cancer stem cells by highthroughput screening. Cell. 2009;138:645-59.

42. Li X, Lewis MT, Huang J, Gutierrez C, Osborne CK, Wu M-F, et al. Intrinsic resistance of tumorigenic breast cancer cells to chemotherapy. JNCI J Natl Cancer Inst. 2008;100:672-9.

43. Perazzoli G, Prados J, Ortiz R, Caba O, Cabeza L, Berdasco M, et al. Temozolomide resistance in glioblastoma cell lines: implication of MGMT, MMR, P-glycoprotein and CD133 expression. Alonso MM, editor. PLOS ONE. 2015:10:e0140131.
44. Bao S, Wu Q, McLendon RE, Hao Y, Shi Q, Hjelmeland AB, et al. Glioma stem cells promote radioresistance by preferential activation of the DNA damage response. Nature. 2006;444:756-60.

45. Auffinger B, Tobias AL, Han Y, Lee G, Guo D, Dey M, et al. Conversion of differentiated cancer cells into cancer stem-like cells in a glioblastoma model after primary chemotherapy. Cell Death Differ. 2014;21:1119-31.

46. Jia B, Liu W, Gu J, Wang J, Lv W, Zhang W, et al. MiR-7-5p suppresses stemness and enhances temozolomide sensitivity of drug-resistant glioblastoma cells by targeting Yin Yang 1. Exp Cell Res. 2019;375:73-81.

47. Liebelt BD, Shingu T, Zhou X, Ren J, Shin SA, Hu J. Glioma stem cells: signaling, microenvironment, and therapy. Stem Cells Int. 2016;2016:1-10.

48. Lin B, Lee H, Yoon J-G, Madan A, Wayner E, Tonning S, et al. Global analysis of H3K4me3 and H3K27me3 profiles in glioblastoma stem cells and identification of SLC17A7 as a bivalent tumor suppressor gene. Oncotarget [Internet]. 2015 [cited 2019 Jul 23];6. http://www.oncotarget.com/fullt ext/3030

49. Hall AW, Battenhouse AM, Shivram H, Morris AR, Cowperthwaite MC, Shpak M, et al. Bivalent chromatin domains in glioblastoma reveal a subtype-specific signature of glioma stem cells. Cancer Res. 2018;78:2463-74.

50. Mack SC, Singh I, Wang X, Hirsch R, Wu Q, Villagomez R, et al. Chromatin landscapes reveal developmentally encoded transcriptional states that define human glioblastoma. J Exp Med. 2019;jem.20190196.

51. Johnston MJ, Nikolic A, Ninkovic N, Guilhamon P, Cavalli FMG, Seaman $S$, et al. High-resolution structural genomics reveals new therapeutic vulnerabilities in glioblastoma. Genome Res. 2019;29:1211-22.

52. Pękowska A, Klaus B, Xiang W, Severino J, Daigle N, Klein FA, et al. Gain of CTCF-anchored chromatin loops marks the exit from naive pluripotency. Cell Syst. 2018;7:482-495.e10.

53. Mallm J, Windisch P, Biran A, Gal Z, Schumacher S, Glass R, et al. Glioblastoma initiating cells are sensitive to histone demethylase inhibition due to epigenetic deregulation. Int J Cancer. 2019;ijc.32649.

54. Modrek AS, Golub D, Khan T, Bready D, Prado J, Bowman C, et al. Lowgrade astrocytoma mutations in IDH1, P53, and ATRX cooperate to block differentiation of human neural stem cells via repression of SOX2. Cell Rep. 2017;21:1267-80.

55. Chinnaiyan P, Vallabhaneni G, Armstrong E, Huang S-M, Harari PM. Modulation of radiation response by histone deacetylase inhibition. Int J Radiat Oncol Biol Phys. 2005;62:223-9.

56. Marks PA, Xu W-S. Histone deacetylase inhibitors: potential in cancer therapy. J Cell Biochem. 2009;107:600-8.

57. Diss E, Nalabothula N, Nguyen D, Chang E, Kwok Y, Carrier F. VorinostatSAHA promotes hyper-radiosensitivity in wild type p53 human glioblastoma cells. J Clin Oncol Res. 2014;2.

58. Wang Q, Jia S, Wang D, Chen X, Kalvakolanu DV, Zheng H, et al. A combination of BRD4 and HDAC3 inhibitors synergistically suppresses glioma stem cell growth by blocking GLI1/L-6/STAT3 signaling axis. Mol Cancer Ther. 2020;

59. Kitange GJ, Mladek AC, Carlson BL, Schroeder MA, Pokorny JL, Cen L, et al. Inhibition of histone deacetylation potentiates the evolution of acquired temozolomide resistance linked to MGMT upregulation in glioblastoma xenografts. Clin Cancer Res. 2012;18:4070-9.

60. Berdasco M, Ropero S, Setien F, Fraga MF, Lapunzina P, Losson R, et al. Epigenetic inactivation of the Sotos overgrowth syndrome gene histone methyltransferase NSD1 in human neuroblastoma and glioma. Proc Natl Acad Sci. 2009;106:21830-5.

61. Was H, Krol SK, Rotili D, Mai A, Wojtas B, Kaminska B, et al. Histone deacetylase inhibitors exert anti-tumor effects on human adherent and stem-like glioma cells. Clin Epigenetics. 2019;11:11.

62. Knoechel B, Roderick JE, Williamson KE, Zhu J, Lohr JG, Cotton MJ, et al. An epigenetic mechanism of resistance to targeted therapy in $T$ cell acute lymphoblastic leukemia. Nat Genet. 2014;46:364-70.

63. Lovén J, Hoke HA, Lin CY, Lau A, Orlando DA, Vakoc CR, et al. Selective inhibition of tumor oncogenes by disruption of super-enhancers. Cell. 2013;153:320-34

64. Berenguer-Daizé C, Astorgues-Xerri L, Odore E, Cayol M, Cvitkovic E, Noel $\mathrm{K}$, et al. OTX015 (MK-8628), a novel BET inhibitor, displays in vitro and in vivo antitumor effects alone and in combination with conventional therapies in glioblastoma models. Int J Cancer. 2016;139:2047-55.

65. Jing D, Huang Y, Liu X, Sia KCS, Zhang JC, Tai X, et al. Lymphocyte-specific chromatin accessibility pre-determines glucocorticoid resistance in acute lymphoblastic leukemia. Cancer Cell. 2018;34:906-921.e8. 
66. Gasparian AV, Burkhart CA, Purmal AA, Brodsky L, Pal M, Saranadasa M, et al. Curaxins: anticancer compounds that simultaneously suppress NF-B and activate p53 by targeting FACT. Sci Transl Med. 2011;3:95ra74-95ra74.

67. Kantidze OL, Luzhin AV, Nizovtseva EV, Safina A, Valieva ME, Golov AK, et al. The anti-cancer drugs curaxins target spatial genome organization. Nat Commun. 2019;10:1441.

68. Tarjan DR, Flavahan WA, Bernstein BE. Epigenome editing strategies for the functional annotation of CTCF insulators. Nat Commun. 2019;10:4258.

69. Hegi ME, Diserens A-C, Gorlia T, Hamou M-F, de Tribolet N, Weller M, et al. MGMT gene silencing and benefit from temozolomide in glioblastoma. N Engl J Med. 2005;352:997-1003.

70. Noushmehr H, Weisenberger DJ, Diefes K, Phillips HS, Pujara K, Berman BP, et al. Identification of a CpG island methylator phenotype that defines a distinct subgroup of glioma. Cancer Cell. 2010;17:510-22.

71. Park JH, de Lomana ALG, Marzese DM, Juarez T, Feroze A, Hothi P, et al. A systems approach to brain tumor treatment. Cancers. 2021;13:3152.

72. Spektor R, Tippens ND, Mimoso CA, Soloway PD. methyl-ATAC-seq measures DNA methylation at accessible chromatin. Genome Res. 2019;29:969-77

73. Barnett KR, Decato BE, Scott TJ, Hansen TJ, Chen B, Attalla J, et al. ATAC-Me captures prolonged DNA methylation of dynamic chromatin accessibility loci during cell fate transitions. Mol Cell. 2020;77:1350-1364.e6.

74. Giesselmann P, Brändl B, Raimondeau E, Bowen R, Rohrandt C, Tandon R, et al. Analysis of short tandem repeat expansions and their methylation state with nanopore sequencing. Nat Biotechnol. 2019;37:1478-81.

75. Liu Q, Fang L, Yu G, Wang D, Xiao C-L, Wang K. Detection of DNA base modifications by deep recurrent neural network on Oxford nanopore sequencing data. Nat Commun. 2019;10:2449.

76. Ni P, Huang N, Zhang Z, Wang D-P, Liang F, Miao Y, et al. DeepSignal: detecting DNA methylation state from Nanopore sequencing reads using deep-learning. Bioinforma Oxf Engl. 2019:35:4586-95.

77. Kempfer R, Pombo A. Methods for mapping 3D chromosome architecture. Nat Rev Genet. 2020;21:207-26.

78. Beagrie RA, Scialdone A, Schueler M, Kraemer DCA, Chotalia M, Xie SQ, et al. Complex multi-enhancer contacts captured by genome architecture mapping. Nature. 2017;543:519-24.
79. Javierre BM, Burren OS, Wilder SP, Kreuzhuber R, Hill SM, Sewitz S, et al. Lineage-specific genome architecture links enhancers and non-coding disease variants to target gene promoters. Cell. 2016;167:1369-1384.e19.

80. Wang X, Tucker NR, Rizki G, Mills R, Krijger PH, de Wit E, et al. Discovery and validation of sub-threshold genome-wide association study loci using epigenomic signatures. eLife. 2016;5

81. Bell RJA, Rube HT, Xavier-Magalhães A, Costa BM, Mancini A, Song JS, et al. Understanding TERT promoter mutations: a common path to immortality. Mol Cancer Res MCR. 2016;14:315-23.

82. Bell RJA, Rube HT, Kreig A, Mancini A, Fouse SD, Nagarajan RP, et al. Cancer. The transcription factor GABP selectively binds and activates the mutant TERT promoter in cancer. Science. 2015;348:1036-9.

83. Eckel-Passow JE, Lachance DH, Molinaro AM, Walsh KM, Decker PA,

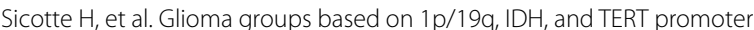
mutations in tumors. N Engl J Med. 2015;372:2499-508.

84. Plaisier CL, O'Brien S, Bernard B, Reynolds S, Simon Z, Toledo CM, et al. Causal mechanistic regulatory network for glioblastoma deciphered using systems genetics network analysis. Cell Syst. 2016;3:172-86.

85. Zlochower A, Chow DS, Chang P, Khatri D, Boockvar JA, Filippi CG. Deep learning $\mathrm{Al}$ applications in the imaging of glioma. Top Magn Reson Imaging TMRI. 2020;29:115-110.

86. Jeong J, Wang L, Ji B, Lei Y, Ali A, Liu T, et al. Machine-learning based classification of glioblastoma using delta-radiomic features derived from dynamic susceptibility contrast enhanced magnetic resonance images: Introduction. Quant Imaging Med Surg. 2019;9:1201-13.

87. Booth TC, Williams M, Luis A, Cardoso J, Ashkan K, Shuaib H. Machine learning and glioma imaging biomarkers. Clin Radiol. 2020;75:20-32.

88. Sotoudeh H, Shafaat O, Bernstock JD, Brooks MD, Elsayed GA, Chen JA, et al. Artificial intelligence in the management of glioma: era of personalized medicine. Front Oncol. 2019;9:768.

\section{Publisher's Note}

Springer Nature remains neutral with regard to jurisdictional claims in published maps and institutional affiliations.
Ready to submit your research? Choose BMC and benefit from:

- fast, convenient online submission

- thorough peer review by experienced researchers in your field

- rapid publication on acceptance

- support for research data, including large and complex data types

- gold Open Access which fosters wider collaboration and increased citations

- maximum visibility for your research: over 100M website views per year

At BMC, research is always in progress.

Learn more biomedcentral.com/submissions 